\title{
Prevenção do tromboembolismo venoso em cirurgia plástica: uma revisão de
}

\section{literatura}

\author{
Prevention of venous thromboembolism in plastic surgery: a literature review \\ Prevención del tromboembolismo venoso en cirugía plástica: revisión de la literatura
}

Recebido: 21/08/2021 | Revisado: 28/08/2021 | Aceito: 28/08/2021 | Publicado: 31/08/2021

Laila Caroline Silva Sousa

ORCID: https://orcid.org/0000-0002-9632-8208

Centro Universitário de Patos de Minas, Brasil

E-mail: lailacarolline23@hotmail.com

Ana Flávia Silva

ORCID: https://orcid.org/0000-0002-8248-3143

Centro Universitário de Patos de Minas, Brasil E-mail: anaflaviasilva@unipam.edu.br

Bárbara Queiroz de Figueiredo

ORCID: https://orcid.org/0000-0003-1630-4597

Centro Universitário de Patos de Minas, Brasil

E-mail: barbarafigueiredo@unipam.edu.br

Bruno Faria Coury

ORCID: https://orcid.org/0000-0002-9236-8552

Centro Universitário de Patos de Minas, Brasil

E-mail: brunofaria@unipam.edu.br

Felipe Alves Soares

ORCID: https://orcid.org/0000-0001-9528-7048

Centro Universitário de Patos de Minas, Brasil

E-mail: felipealves@unipam.edu.br

Natália Caroline Caixeta

ORCID: https://orcid.org/0000-0001-7547-2510

Centro Universitário de Patos de Minas, Brasil E-mail: nataliacaixeta@unipam.edu.br

Nicolly Skarlet Souto Oliveira

ORCID: https://orcid.org/0000-0003-3304-9946

Centro Universitário de Patos de Minas, Brasil

E-mail: nicollyskarlet@gmail.com

Rafael Sávio Soares

ORCID: https://orcid.org/0000-0002-9469-9558

Hospital Nossa Senhora de Fátima, Brasil

E-mail: rafaelsavio@hnsf.com.br

\begin{abstract}
Resumo
Introdução: O tromboembolismo venoso é uma complicação que acontece em cirurgias plásticas e pode ser potencialmente grave. Objetivo: revisar estudos e evidências relacionados à protocolos de prevenção do tromboembolismo venoso na área da cirurgia plástica. Metodologia: pesquisa descritiva do tipo revisão integrativa da literatura, realizada no mês de julho de 2021, através do acesso online nas bases de dados National Library of Medicine (PubMed MEDLINE), Scientific Electronic Library Online (Scielo), Cochrane Database of Systematic Reviews (CDSR), Google Scholar, Biblioteca Virtual em Saúde (BVS) e EBSCO Information Services. Resultados: A partir da revisão integrativa da literatura, observa-se que existem controvérsias entre estudos, ou seja, ainda não há um consenso entre autores a respeito da profilaxia para tromboembolismo venoso em cirurgia plástica. Alguns autores reiteram que pacientes hígidos e sem histórico de comorbidades não possuem necessidade de iniciar profilaxia para TEV. No entanto, há estudiosos que ressaltam o risco expressivo do desenvolvimento de um TEV na cirurgia plástica, principalmente em processos cirúrgicos que apresentem determinantes como tempo de operação acima de 2 horas, tipo de decúbito e procedimento, além de cirurgias combinadas. Nesse sentido, há estudos que defendem o uso de quimioprofiláticos, como Enoxaparina e Rivaroxabana, conforme a singularidade do indivíduo e do procedimento, visando, sobretudo, preservar a saúde do paciente. Conclusão: Diante dos achados, a tromboprofilaxia é a melhor estratégia de prevenção em pacientes cirúrgicos, considerando que cada caso é único, e o risco de tromboembolismo venoso depende tanto da condição do paciente, quanto do procedimento realizado.
\end{abstract}

Palavras-chave: Tromboembolismo venoso; Cirurgia plástica; Trombose; Pré-operatório; Protocolo. 


\begin{abstract}
Introduction: Venous thromboembolism is a complication that occurs in plastic surgery and can be potentially serious. Objective: to review studies and evidence related to prevention protocols for venous thromboembolism in the field of plastic surgery. Methodology: descriptive research of the integrative literature review type, carried out in July 2021, through online access in the National Library of Medicine (PubMed MEDLINE), Scientific Electronic Library Online (Scielo), Cochrane Database of Systematic Reviews (CDSR), Google Scholar, Virtual Health Library (VHL) and EBSCO Information Services. Results: From the comprehensive review of the literature, it is observed that there are controversies between studies, that is, there is still no consensus among authors regarding prophylaxis for venous thromboembolism in plastic surgery. Some authors reiterate that healthy patients with no history of comorbidities do not need to start prophylaxis for VTE. However, there are scholars who emphasize the significant risk of developing a VTE in plastic surgery, especially in surgical procedures that present determinants such as operating time above 2 hours, type of decubitus and procedure, in addition to combined surgeries. In this sense, there are studies that support the use of chemoprophylactics, such as enoxaparin and rivaroxaban, according to the uniqueness of the individual and the procedure, aiming, above all, to preserve the patient's health. Conclusion: Based on these findings, thromboprophylaxis is the best prevention strategy in surgical patients, considering that each case is unique, and the risk of venous thromboembolism depends both on the patient's condition and on the procedure performed.
\end{abstract}

Keywords: Venous thromboembolism; Plastic surgery; Thrombosis; Preoperative; Protocol.

\title{
Resumen
}

Introducción: El tromboembolismo venoso es una complicación que se presenta en la cirugía plástica y puede ser potencialmente grave. Objetivo: revisar los estudios y la evidencia relacionados con los protocolos de prevención de la tromboembolia venosa en el campo de la cirugía plástica. Metodología: investigación descriptiva del tipo revisión integradora de la literatura, realizada en julio de 2021, mediante acceso online en la Biblioteca Nacional de Medicina (PubMed MEDLINE), Scientific Electronic Library Online (Scielo), Cochrane Database of Systematic Reviews (CDSR), Google Scholar, Biblioteca Virtual en Salud (BVS) y Servicios de Información EBSCO. Resultados: De la revisión exhaustiva de la literatura se observa que existen controversias entre estudios, es decir, aún no existe consenso entre los autores en cuanto a la profilaxis del tromboembolismo venoso en cirugía plástica. Algunos autores reiteran que los pacientes sanos sin antecedentes de comorbilidades no necesitan iniciar profilaxis para TEV. Sin embargo, hay estudiosos que enfatizan el riesgo significativo de desarrollar un TEV en cirugía plástica, especialmente en procedimientos quirúrgicos que presentan determinantes como tiempo operatorio superior a 2 horas, tipo de decúbito y procedimiento, además de cirugías combinadas. En este sentido, existen estudios que avalan el uso de quimioprofilácticos, como enoxaparina y rivaroxabán, según la singularidad del individuo y del procedimiento, con el objetivo, sobre todo, de preservar la salud del paciente. Conclusión: Con base en estos hallazgos, la tromboprofilaxis es la mejor estrategia de prevención en pacientes quirúrgicos, considerando que cada caso es único, y el riesgo de tromboembolismo venoso depende tanto de la condición del paciente como del procedimiento realizado.

Palabras clave: Tromboembolismo venoso; Cirugía plástica; Trombosis; Preoperatorio; Protocolo.

\section{Introdução}

A entidade nosológica conhecida como tromboembolismo venoso (TEV) possui um grande aspecto de apresentação clínica, sendo a trombose venosa profunda (TVP) e o tromboembolismo pulmonar (TEP) seus principais componentes, representando, assim, complicações frequentes para pacientes submetidos a cirurgias (Justino et al. 2018). Desse modo, a cirurgia por si só se configura como um importante fator gerador do tromboembolismo venoso, porém deve-se pensar em diversos fatores de risco associados ao paciente, como a idade superior a 60 anos e casos de obesidade (IMC>30), além das condições de predisposição, como hipovolemia, restrição dos movimentos, estase sanguínea e trauma dos tecidos (Paiva et al., 2015).

Nesse sentido, a trombose venosa profunda e o tromboembolismo pulmonar são muitas vezes clinicamente silenciosos e com isso, tornam-se de difícil diagnóstico. Além disso, eles podem ser considerados como oportunistas, já que por apresentarem uma baixa incidência, muitas vezes o cirurgião plástico não aplica protocolos de prevenção, tanto farmacológicos, como a administração de anticoagulantes, quanto não farmacológicos, como o uso de meias elásticas e a deambulação precoce (Reis Neto et al., 2019). Associado a isso, ainda há uma grande resistência na adoção de protocolos baseados na quimioprofilaxia, como por exemplo, o uso da heparina de baixo peso molecular, devido ao risco do aumento de casos de sangramentos e outras complicações (Paiva et al., 2015). 
Quando sintomática, a trombose venosa profunda pode apresentar-se por dor à palpação na panturrilha ou à dorsiflexão dos pés, sinal de Homans positivo e edema da área acometida. Essa manifestação pode agravar-se, dando sinais como palidez, taquicardia e hipotensão, podendo alcançar um desfecho mais grave, a própria embolia pulmonar (Santos et al. 2017). Nesse sentido, segundo Justino, Varoni e Duz (2018), apesar de existirem poucas publicações sobre a incidência de tromboembolismo venoso na cirurgia plástica, acredita-se que ela sofre variação de acordo com o tipo de cirurgia realizada. Dessa forma, considera-se aproximadamente $0,35 \%$ para lifting facial, 1,3\% em casos de reconstrução de mama, 9,4\% na lipoaspiração circunferencial, 6,6\% para abdominoplastia combinada a outro procedimento em comparação com 1,4 a $2 \%$ para abdominoplastias realizadas isoladamente (Reis Neto et al., 2019).

O número de cirurgias plásticas realizadas anualmente, bem como a associação de procedimentos, sofre um aumento substancial, logo, a possibilidade de complicações também cresce proporcionalmente. Dessa maneira, eventos tromboembólicos têm sido alvo de maior pesquisa e atenção, visando um aumento na segurança dos pacientes (Valente et al., 2014). Sob essa perspectiva, esse estudo tem como objetivo investigar, por meio da revisão literária, os principais protocolos aplicados na prevenção do tromboembolismo venoso dentro da cirurgia plástica.

\section{Metodologia}

Trata-se de uma pesquisa descritiva do tipo revisão integrativa da literatura, que buscou responder quais as evidências sobre o tromboembolismo venoso em cirurgia plástica, com foco nas formas preventivas. A pesquisa foi realizada através do acesso online nas bases de dados National Library of Medicine (PubMed MEDLINE), Scientific Electronic Library Online (Scielo), Cochrane Database of Systematic Reviews (CDSR), Google Scholar, Biblioteca Virtual em Saúde (BVS) e EBSCO Information Services, no mês de julho de 2021. Para a busca das obras foram utilizadas as palavras-chaves presentes nos descritores em Ciências da Saúde (DeCS): em inglês: "venous thromboembolism", "thrombosis", "plastic surgery", "protocol", "preoperative care", em português: "tromboembolismo venoso", " trombose", "cirurgia plástica", "protocolo", "cuidados préoperatórios", e em espanhol: "tromboembolismo venoso", "trombosis", "cirugía plástica", "protocolo", "cuidados preoperatorios"

Como critérios de inclusão, foram considerados artigos originais, que abordassem o tema pesquisado e permitissem acesso integral ao conteúdo do estudo, publicados no período de 2003 a 2021, em inglês, espanhol e português. O critério de exclusão foi imposto naqueles trabalhos que não estavam em inglês ou português, que não tinham passado por processo de Peer-View e que não abordassem o tromboembolismo venoso em cirurgia plástica. A estratégia de seleção dos artigos seguiu as seguintes etapas: busca nas bases de dados selecionadas; leitura dos títulos de todos os artigos encontrados e exclusão daqueles que não abordavam o assunto; leitura crítica dos resumos dos artigos e leitura na íntegra dos artigos selecionados nas etapas anteriores. Assim, totalizaram-se 22 artigos científicos para a revisão integrativa da literatura, com os descritores apresentados acima. Após esta seleção, filtraram-se por artigos dos últimos dezoito anos e por artigos em línguas portuguesa, espanhola e inglesa.

\section{Resultados e Discussão}

A partir da análise final da íntegra dos 22 artigos devidamente selecionados, atendendo às variáveis e aos objetivos desse estudo, foi possível observar que muitos há muitas controversias e que ainda não há um consenso a respeito da profilaxia para tromboembolismo venoso em cirurgia plástica. A Tabela 1 a seguir foi construída de modo a simplificar as principais informações e proporcionar melhor visualização de cada estudo utilizado. 
Tabela 1. Conteúdo dos artigos incluídos na revisão.

\begin{tabular}{|c|c|c|}
\hline ESTUDO & TÍTULO & ACHADOS PRINCIPAIS \\
\hline Valente et al., 2014 & $\begin{array}{l}\text { Venous Thromboembolism following } \\
\text { Elective Aesthetic Plastic Surgery: A } \\
\text { Longitudinal Prospective Study in } 1254 \\
\text { Patients }\end{array}$ & $\begin{array}{l}\text { Tabagismo, duas ou mais gestações, além de reposição hormonal são fatores de } \\
\text { risco para aumento de TEV. Não foram encontradas nenhuma correlação entre } \\
\text { mastopexia, mamoplastia de aumento, rinoplastia e lipoaspiração como fatores de } \\
\text { risco isolados para TEV. Não a consenso sobre uso de profilaxia em pacientes } \\
\text { previamente saudáveis que irão realizar cirurgias estéticas eletivas. Duas ou mais } \\
\text { horas de cirurgias, bem como uso de anestesia não foram associados ao aumento } \\
\text { de TEV nesse estudo. }\end{array}$ \\
\hline Iorio et al., 2015 & $\begin{array}{l}\text { Practical Guidelines for Venous } \\
\text { Thromboembolism Chemoprophylaxis } \\
\text { in Elective Plastic Surgery }\end{array}$ & $\begin{array}{l}\text { Aborda sobre o risco de hematomas poder superar os benefícios no uso de } \\
\text { quimioprofilaxia em cirurgias plásticas eletivas. O risco de tromboembolismo } \\
\text { venoso vai depender da variante paciente e do procedimento. Uso de } \\
\text { quimioprofilaxia em doses baixas não apresenta tantos riscos de sangramentos no } \\
\text { pós-operatório. }\end{array}$ \\
\hline Drouin et al., 2018 & $\begin{array}{l}\text { Épidémiologie des } \quad \text { thromboses } \\
\text { veineuses des membres } \\
\text { étude rérieurs: } \\
\text { aiguës }\end{array}$ & $\begin{array}{l}\text { Pacientes com comorbidades graves, que utilizam cateteres venosos centrais, } \\
\text { possuem maior chance de desenvolver trombose venosa em membro superior. }\end{array}$ \\
\hline Almeida et al. 2018 & $\begin{array}{l}\text { Assistência da enfermagem na trombose } \\
\text { venosa profunda }\end{array}$ & $\begin{array}{l}\text { A assistência de enfermagem não possui informação suficiente sobre trombose } \\
\text { venosa profunda bem como, suas consequências e dever para com paciente. }\end{array}$ \\
\hline Amaral et al. 2017 & $\begin{array}{l}\text { Estudo Tromboembolismo Venoso pós- } \\
\text { Operatório (TREVO) - risco e } \\
\text { mortalidade por especialidade cirúrgica }\end{array}$ & $\begin{array}{l}\text { O risco de tromboembolismo venoso sintomático pós-operatório foi de 1,33/1000. } \\
\text { A especialidade com menor risco foi a cirurgia plástica. }\end{array}$ \\
\hline Paiva et al. 2010 & $\begin{array}{l}\text { Tromboembolismo venoso em Cirurgia } \\
\text { Plástica: protocolo de prevenção na } \\
\text { clínica Ivo Pitanguy }\end{array}$ & $\begin{array}{l}\text { Não houve casos de hematomas e TEV em nenhum dos } 711 \text { pacientes operados no } \\
\text { período do estudo, após implementação do protocolo de prevenção. As cirurgias } \\
\text { combinadas têm sido demonstradas como de maior risco para fenômenos } \\
\text { tromboembólicos, com destaque aquelas associadas à abdominoplastia ou à } \\
\text { lipoaspiração. }\end{array}$ \\
\hline Santos et al. 2017 & $\begin{array}{l}\text { Os Entraves da Profilaxia da Trombose } \\
\text { Venosa Profunda: Uma Revisão } \\
\text { Integrativa da Literatura }\end{array}$ & $\begin{array}{l}\text { A trombose Venosa Profunda (TVP) está relacionada com o desabastecimento da } \\
\text { medicação a ser fornecido de forma gratuita, a desinformação e a falta de preparo } \\
\text { para administração do anticoagulante pelo paciente. Há muita divergência ainda } \\
\text { quanto ao momento de se iniciar anticoagulação profilática, se antes ou após, o ato } \\
\text { cirúrgico. A orientação multiprofissional, por parte do enfermeiro e farmacêutico } \\
\text { a respeito das orientações medicamentosas pós-alta hospitalar pode reduzir a TVP. }\end{array}$ \\
\hline Roça et al. 2019 & $\begin{array}{l}\text { Rivaroxabana para profilaxia } \\
\text { tromboembolismo venoso } \\
\text { abdominoplastia após grande } \\
\text { ponderal: } 396 \text { casos. }\end{array}$ & $\begin{array}{l}\text { A profilaxia para TEV é recomendada pelo menos } 10 \text { dias antes e pós } \\
\text { procedimento de alto risco. A rivaroxabana em pacientes submetidos } \\
\text { a abdominoplastia tem baixa incidência de TEV. }\end{array}$ \\
\hline Swanson, 2017 & $\begin{array}{l}\text { Concerns Regarding the Use of OralAnt } \\
\text { icoagulants (Rivaroxaban and Apixaban } \\
\text { )for Venous Thromboembolism Prophyl } \\
\text { axis in Plastic Surgery Patients }\end{array}$ & A rivaroxabana provocou uma taxa de $2,8 \%$ de hematomas nos pacientes. \\
\hline Barros, 2021 & $\begin{array}{l}\text { Prevenção de tromboembolismo venoso } \\
\text { em cirurgia plástica: revisão de } \\
\text { literatura. }\end{array}$ & $\begin{array}{l}\text { Houve necessidade de reoperação em casos de uso da Rivaroxabana. O uso de } \\
\text { enoxaparina no pós operatório não aumentou a taxa de reoperação e hematoma } \\
\text { significativamente, mas houve sangramento em operações de grandes áreas de } \\
\text { dissecação e áreas altamente vascularizadas. }\end{array}$ \\
\hline Anger et al. 2003 & $\begin{array}{ll}\text { Um Protocolo de } & \text { Prevenção de } \\
\text { Trombose Venosa } & \text { Profunda } \\
\text { Cirurgia Plástica } & \end{array}$ & $\begin{array}{l}\text { Relata que em } 1999 \text { não havia um protocolo que abordasse o risco de TVP em } \\
\text { cirurgia plástica. Determinadas cirurgias ou condutas são específicas da Cirurgia } \\
\text { Plástica e aumentam o risco da TVP, devido ao tipo de decúbito, prazo de cirurgia } \\
\text { e limitações do pós-operatório. Outros riscos incluem o uso de anticoncepcionais } \\
\text { e terapia de reposição hormonal. }\end{array}$ \\
\hline Abreu et al. 2016 & $\begin{array}{l}\text { Risco tromboembólico: etiopatogenia e } \\
\text { profilaxia em pacientes } \\
\text { mastectomizadas submetidas a } \\
\text { reconstrução mamária }\end{array}$ & $\begin{array}{l}\text { Apesar da profilaxia ser considerada como segura e efetiva, a mesma permanece } \\
\text { subutilizada. A avaliação do risco de TEV por uma estratificação de risco } \\
\text { individual ponderado corresponde a um dos métodos preferidos e cientificamente } \\
\text { mais bem comprovados pela literatura. Entre procedimentos de cirúrgica plástica, } \\
\text { o risco de TEP sintomático é maior na lipoaspiração, com uma incidência máxima }\end{array}$ \\
\hline
\end{tabular}




\begin{tabular}{|c|c|c|}
\hline & & $\begin{array}{l}\text { registada de } 23,0 \% \text {. A reconstrução mamária é o segundo procedimento, com uma } \\
\text { incidência máxima de } 6,0 \% \text {, seguida por lesões térmicas }(4,4 \%) \text {, abdominoplastia } \\
(0,3 \text { a } 3,4 \%) \text { e reconstrução oncológica da cabeça e pescoço }(0,1 \text { a } 0,4 \%) \text {. Dentro } \\
\text { da cirurgia da mama, o risco de TEV é influenciado pelo tipo de cirurgia mamária, } \\
\text { mais especificamente pela adição de reconstrução mamária, quando comparada } \\
\text { com outros tipos de cirurgia mamária. }\end{array}$ \\
\hline Hinrichsen et al. 2014 & $\begin{array}{l}\text { Implementação do programa de } \\
\text { prevenção de Tromboembolismo } \\
\text { Venoso (TEV): Estudo Piloto. }\end{array}$ & $\begin{array}{l}\text { As diretrizes para TEV são complexas e dependem da adesão às estratégias } \\
\text { profiláticas realizadas por meio de protocolos. Ainda há falta de sistematização na } \\
\text { avaliação de riscos para tromboembolismo, seja em pacientes clínicos e ou } \\
\text { cirúrgicos. Entre as situações mais comuns em que a profilaxia tem sido } \\
\text { inadequada a cirurgia plástica é uma delas. A profilaxia do TEV é singular e feita } \\
\text { por medidas farmacológicas ou mecânicas. Após a alta hospitalar, a prevenção } \\
\text { deve persistir entre os que ainda sinalizem alguns riscos. O uso indevido de } \\
\text { quimioprofiláticos pode aumentar riscos, especialmente de sangramento. }\end{array}$ \\
\hline Reis Neto et al. 2019 & $\begin{array}{l}\text { Fenômenos tromboembólicos } \\
\text { associados a mamoplastia no Serviço de } \\
\text { Cirurgia Plástica do Hospital PUC- } \\
\text { Campinas }\end{array}$ & $\begin{array}{l}\text { A mamoplastia apresenta-se como um fator de risco para o desenvolvimento de } \\
\text { tromboembolismo, principalmente, em razão da estase venosa, que é agravada } \\
\text { pela imobilização prolongada na mesa cirúrgica. Tanto a profilaxia mecânica, que } \\
\text { envolve a compressão intermitente das panturrilhas e a meia elástica, quanto a } \\
\text { química, que se constitui na heparinoterapia, apresentam eficácia contra o } \\
\text { tromboembolismo venoso (TEV). }\end{array}$ \\
\hline Justino et al. 2017 & $\begin{array}{l}\text { Tromboembolismo venoso (TEV) em } \\
\text { abdominoplastias: um protocolo de } \\
\text { prevenção }\end{array}$ & $\begin{array}{l}\text { A abdominoplastia apresenta diversos fatores que aumentam o risco de eventos } \\
\text { tromboembólicos como: lesão de vasos, com intensa dissecação e rompimento de } \\
\text { veias superficiais; tempo operatório longo; anestesia geral, com diminuição da } \\
\text { resistência vascular periférica; posição do paciente no intraoperatório que acarreta } \\
\text { a redução do retorno venoso das extremidades (estase venosa); mobilidade } \\
\text { reduzida no pós-operatório; obesidade e plicatura da diástase do músculo reto } \\
\text { abdominal, o que diminui o retorno venoso. Abdominoplastia isolada (não } \\
\text { combinada a nenhum outro procedimento) está associada a uma taxa de } 0,1 \% \text { a } \\
1,1 \% \text { de TVP, } 0,34 \% \text { a } 1,1 \% \text { de TEP e } 0,5 \text { a } 8 \% \text { de hematoma, sendo que quando } \\
\text { é realizada cirurgia combinada esses valores aumentam significativamente. } \\
\text { Considerando que a cascata de coagulação é ativada já durante a cirurgia } \\
\text { (abdominoplastia), seria interessante iniciar a profilaxia no pré-operatório a fim de } \\
\text { evitar a ativação da mesma. }\end{array}$ \\
\hline Mittal et al. 2020 & $\begin{array}{l}\text { Venous Thromboembolism (VTE) } \\
\text { Prophylaxis After Abdominoplasty and } \\
\text { Liposuction: A Review of the Literature }\end{array}$ & $\begin{array}{l}\text { A abdominoplastia é a cirurgia estética mais relacionada a casos de } \\
\text { tromboembolismo, sendo que, se associada a outros procedimentos na região } \\
\text { abdominal, como lipoaspiração, a sua incidência aumenta. O surgimento de } \\
\text { hematoma pós cirúrgico está diretamente relacionado com o nível de invasão do } \\
\text { procedimento realizado. }\end{array}$ \\
\hline Paiva et al. 2015 & $\begin{array}{l}\text { Protocolo de prevenção de } \\
\text { tromboembolismo venoso em cirurgia } \\
\text { plástica: resultados em } 2759 \text { pacientes } \\
\text { no Instituto Ivo Pitanguy }\end{array}$ & $\begin{array}{l}\text { A crença atual de que o risco de TVE é baixo em cirurgia plástica é enganosa. } \\
\text { Existe grande dificuldade em reconhecer a doença, que pode assumir proporções } \\
\text { catastróficas tais como tromboembolismo pulmonar fatal. Infelizmente, este pode } \\
\text { ser a primeira e a única manifestação da doença. Na literatura a taxa de TEV em } \\
\text { mamoplastia é estabelecida como } 0,23 \% \text { e em abdominoplastias como } 1,16 \% \text {. } \\
\text { Não encontramos dados na literatura em relação às taxas de TEV em rinoplastia } \\
\text { reconstrutiva. Entre os } 34 \text { pacientes que apresentaram hematomas, } 19 \text { casos } \\
(55,9 \%) \text { foram observados após a quimioprofilaxia e } 15 \text { casos }(44,1 \%) \text { ocorreram } \\
\text { sem administração de quimioprofilaxia. Os pacientes recebendo heparina } 12 \text { horas } \\
\text { após a cirurgia foram o grupo mais extenso. O grupo recebendo heparina } 1 \text { hora } \\
\text { antes da cirurgia não apresentaram casos de hematoma. }\end{array}$ \\
\hline Hoyos, 2020 & $\begin{array}{l}\text { Tromboembolismo pulmonar decorrente } \\
\text { de tromboflebites venosas superficiais } \\
\text { em membros superiores pós-cirurgia } \\
\text { plástica mamária estética: relato de } 3 \\
\text { casos }\end{array}$ & $\begin{array}{l}\text { A principal quimioprofilaxia na trombose venosa é realizada com fibrinolíticos } \\
\text { (heparina ou heparina de baixo peso molecular), enquanto na trombose arterial se } \\
\text { fundamenta no uso de antiagregantes plaquetários. A profilaxia mecânica (meias } \\
\text { elásticas, aparelhos pneumáticos intermitentes) diminui a estase e distensão } \\
\text { venosa. O aparelho pneumático tem pequena atividade fibrinolítica. Indica-se } \\
\text { iniciá-lo } 30 \text { minutos antes da indução anestésica até a alta do paciente, em } \\
\text { cirurgias maiores que } 1 \text { hora. No pré-operatório, sugere-se seguir os protocolos de } \\
\text { profilaxia para tromboembolismo. Além desse protocolo, no intraoperatório, } \\
\text { recomenda-se a monitorização constante da posição dos braços, bem como o uso } \\
\text { de meias elásticas, aparelho pneumático nos membros inferiores e } \\
\text { quimioprofilaxia. Já no pós-operatório, é essencial a vigilância dos membros } \\
\text { superiores para evitar o edema excessivo, e pesquisa ativa para tromboflebites. }\end{array}$ \\
\hline
\end{tabular}




\begin{tabular}{|c|c|c|}
\hline Amaral et al. 2014 & $\begin{array}{l}\text { Recomendações Perioperatórias para } \\
\text { Profilaxia do Tromboembolismo } \\
\text { Venoso no Doente Adulto. Consenso } \\
\text { Nacional Multidisciplinar } 2014\end{array}$ & $\begin{array}{l}\text { No período peri-operatório o risco de tromboembolismo está relacionado com } \\
\text { fatores individuais, tipo de cirurgia e de anestesia e tempo de internamento. A } \\
\text { tromboprofilaxia é uma responsabilidade multidisciplinar, deve basear-se na } \\
\text { ponderação dos riscos de tromboembolismo venoso e de hemorragia. A } \\
\text { tromboprofilaxia deve iniciar-se } 6-12 \text { horas após a cirurgia (com exceções). }\end{array}$ \\
\hline Murphy et al. 2012 & $\begin{array}{l}\text { Evidence-Based Practices for } \\
\text { Thromboembolism Prevention: } \\
\text { Summary of the ASPS Venous } \\
\text { Thromboembolism Task Force Report }\end{array}$ & $\begin{array}{l}\text { Em pacientes de cirurgia eletiva, na qual o procedimento é agendado com } \\
\text { antecedência e não é realizado para tratar uma situação de emergência ou } \\
\text { urgência, deve-se considerar o uso de estratégias de redção de risco, tais como: } \\
\text { limitar o tempo na sala de operação, redução de peso, descontinuação da terapia } \\
\text { hormonal de reposição, e mobilização pós-operatória precoce. Em pacientes } \\
\text { submetidos a procedimentos realizados com anestesia de duração de mais de } 60 \\
\text { minutos, tais como: contorno corporal, abdominoplastia, reconstrução mamária, } \\
\text { procedimentos de extremidade inferior, procedimentos de câncer de cabeça / } \\
\text { pescoço; deve-se considerar a possibilidade de usar heparina de baixo peso } \\
\text { molecular (LMWH) ou heparina não fraccionada (UH), no pós-operatório. }\end{array}$ \\
\hline Pannucci, 2017 & $\begin{array}{l}\text { Evidence-Based Recipes for Venous } \\
\text { Thromboembolism Prophylaxis: A } \\
\text { Practical Safety Guide. }\end{array}$ & $\begin{array}{l}\text { Indicou fatores de risco que podem ser relevantes para TEV, tais como: viagens } \\
\text { aéreas ou de carro recentes; plicatura de reto; tipo de anestésico; tempo e extensão } \\
\text { da deambulação precoce. Sugere promover uma análise cuidadosa de risco pré- } \\
\text { operatório em procedimentos eletivos (abordar obesidade, uso de } \\
\text { anticoncepcionais orais, procedimentos operatórios recentes etc.). Modificação do } \\
\text { risco intraoperatório e redução do risco, com medidas como: o planejamento para } \\
\text { limitar o tempo operatório e os procedimentos simultâneos; recorrer à anestesia } \\
\text { não geral, se for possível e apropriado; adotar o uso de dispositivos de compressão } \\
\text { sequencial caso a bomba muscular da panturrilha estiver comprometida; etc. Em } \\
\text { casos de pacientes com alto risco de desenvolver TEV, propõe uma } \\
\text { quimioprofilaxia hospitalar pós-operatória, e sugere que se considere a } \\
\text { possibilidade de profilaxia pós-alta em cirurgia ambulatorial ou hospitalar. }\end{array}$ \\
\hline Cuenca-Pardo, 2019 & $\begin{array}{l}\text { Searching for the Best Way to Assess } \\
\text { the Risk of Thrombosis in Aesthetic } \\
\text { Plastic Surgery; The Role of the } \\
\text { Caprini/Pannucci Score }\end{array}$ & $\begin{array}{l}\text { Ao avaliar a acurácia dos critérios dos estudos de Caprini/Pannucci de avaliação } \\
\text { do risco de TEV, notou uma baixa sensibilidade naqueles classificados como de } \\
\text { baixo risco, na qual muitos pacientes com baixas pontuações nos referidos scores } \\
\text { desenvolveram trombose. Para aumentar sua eficácia e tomá-los como base para a } \\
\text { decisão de cuidados profiláticos, é necessário que se modifique a pontuação e que } \\
\text { se adicione alguns fatores. As modificações a serem feitas devem ser validadas } \\
\text { para demonstrar sua eficácia e consistência. O escore Caprini/Pannucci } \\
\text { modificado pode ser muito útil na estratificação do risco de trombose em } \\
\text { pacientes de cirurgia plástica estética e na implementação de cuidados } \\
\text { profiláticos. }\end{array}$ \\
\hline
\end{tabular}

Fonte: Bases de dados selecionadas pelos autores (2021).

A maioria dos estudos abordam os fatores de risco para o desenvolvimento de tromboembolismo venoso em cirurgias estéticas eletivas, destacando se há ou não eficácia da profilaxia medicamentosa e mecânica para prevenir casos de TEV. Segundo Valente et al., (2014), há fatores que predispõe risco maior para o desenvolvimento de tromboembolismo, tal como tabagismo, mais de duas gestações, uso de terapia de reposição hormonal e de anticoncepcionais. O autor reitera que pacientes saudáveis e sem histórico de comorbidades não possuem necessidade de iniciar profilaxia para TEV, bem como Hinrichsen et al., (2014), que afirma que em cirurgias plásticas a prevenção tem sido inadequada, visto que o uso indevido de profiláticos podem acarretar danos graves, como sangramentos. Em consonância a isso, Seattle et al., 2015, discorre que quimioprofiláticos podem apresentar um risco superior aos benefícios em cirurgias plásticas dependendo do paciente. A exemplo disso, Swanson (2017), relata que o uso do anticoagulante Rivaroxabana provocou hematoma em uma parcela dos pacientes analisados em seu estudo e, Barros, (2021), relatou a necessidade de reoperação em pacientes que fizeram o uso desse medicamento. Já o uso de enoxaparina no pós-operatório para a mesma finalidade e profilaxia de TVP, não aumentou a taxa de reoperação e hematomas significativamente, mas causou sangramentos em operações como abdominoplastia.

Entretanto, há muitas divergências e controvérsias, logo autores como Paiva et al., (2015), afirmam que o risco de tromboembolismo venoso em cirurgia plástica não é baixo, como alguns estudiosos acreditam e, reforçam que o desenvolvimento de um TVE (tromboembolismo venoso) pode ser fatal e grave. Nessa mesma perspectiva de acordo com Anger et al., (2003), a cirurgia plástica possui determinantes, como tempo de operação acima de 2 horas de operação, tipo de 
decúbito e procedimento, que oferecem maior risco de desenvolver TVP. Esse estudioso, ainda, relata que a partir de 1999 com implementação de protocolos de profilaxia para TVP em cirurgia plástica, os casos de trombose nesses procedimentos caíram, indicando a importância da adesão desses protocolos para a saúde do paciente. Inclusive, um estudo realizado por Paiva (2010), na clínica Ivo Pitanguy mostrou que nenhum dos 711 pacientes sofreu TEV após a aplicação do protocolo.

Para a maior parte dos estudiosos a abdominoplastia representa o procedimento de maior risco para trombose profunda. Anita P. A. Abreu (2016), diz que o risco de tromboembolismo pulmonar é maior em pacientes que realizam essa cirurgia estética. Nesse contexto, a explicação para isso seria a "lesão de vasos, com intensa dissecação e rompimento de veias superficiais; tempo operatório longo; anestesia geral, com diminuição da resistência vascular periférica)" (Justino et al. 2017). Porém, o cenário é mais preocupante quando se trata de cirurgias combinadas. Ainda, de acordo com Paiva et al., (2010), procedimentos eletivos combinados com abdominoplastia e lipoaspiração oferecem risco maior de TEV, pois há maior estase venosa, devido à grande área de dessecação de veias. Adiante, a mamoplastia também exige cuidados, ficando atrás apenas das cirurgias estéticas de abdome. Segundo, Anita P. A. Abreu (2016), o tipo de cirurgia mamária influencia diretamente no risco de trombose venosa, sendo então a mamoplastia de aumento e reconstrução o procedimento que apresenta maior gravidade.

Sob essa perspectiva, Amaral et al., (2014), pontua que a tromboprofilaxia é um dever multidisciplinar, ou seja, enfermeiros, farmacêuticos, fisioterapeutas e médicos têm responsabilidade mútua em ponderar os riscos e evitar complicações em seus pacientes. Santos et al., (2017), ilustra isso ao dizer que orientações feitas de forma correta por farmacêuticos e enfermeiros na alta hospitalar podem aumentar a adesão a terapia medicamentosa no pós-operatório e reduzir assim, a incidência de trombose e complicações, como reinternações. Porém, Almeida et al., (2018), em seu estudo observou que boa parte dos profissionais da enfermagem não dominam o conhecimento sobre TEV e consequentemente seus riscos para o paciente.

Desse modo, é importante iniciar um cuidado pré-operatório, peri-operatório e pós-operatório, principalmente, em pacientes com risco elevado para TEV. A própria cascata de coagulação sofre alterações durante os procedimentos. Assim, conforme Hoys et al., (2020), a profilaxia mecânica pode ser feita com o uso de meias de compressa e aparelhos pneumáticos cerca de meia hora antes da anestesia geral ser aplicada, para diminuir a estase venosa no momento da cirurgia. Já no perioperatório é preciso ter atenção com a posição dos membros superiores do paciente, a duração do procedimento e o uso de anticoagulantes e antiplaquetários. Além disso é essencial observar a presença de edema nos membros superiores no pósoperatório. Ademais, Roça et al., 2019, considera que o uso de quimioprofiláticos em pessoas de alto risco, deve iniciar 10 dias antes e 10 dias após a operação. Para ele a Rivaroxabana em pacientes que realizaram abdominoplastia é muito eficiente em prevenir TEV. Por isso, é imprescindível, a máxima de Amaral et al., (2014), sobre o paciente ser uma variante singular em cada procedimento, o que significa que a profilaxia correta depende das suas subjetividades.

\section{Conclusão}

O tromboembolismo venoso é uma complicação que acontece recorrentemente em cirurgias plásticas e essa está diretamente relacionada a morbimortalidade dos pacientes internados, que por sua vez é a principal causa de morte hospitalar que poderia ser evitada. A melhor estratégia de prevenção do tromboembolismo venoso em pacientes cirúrgicos ainda é a tromboprofilaxia, método bastante conhecido, mas muitas vezes subutilizado. Assim, fica evidente a necessidade de difundir e debater mais sobre essa técnica por meio de indicação médica e de recursos didáticos na área, a fim de reduzir esse índice.

Dessa forma, no intuito de melhorar tais taxas de morbimortalidade e de prevenir possíveis complicações, é necessário destinar uma maior atenção às complexidades do tromboembolismo venoso. De acordo com a literatura utilizada, a grande maioria dos autores recomendam de maneira coincidente o uso da profilaxia, grande aliada na redução dos índices de TVP. No que tange a profilaxia mecânica e a quimioprofilaxia, ambas utilizam a heparinoterapia, e essas apresentam uma redução na 
incidência de TVP e devem ser utilizadas baseadas na ponderação de riscos de tromboembolismo e de hemorragias, individuais as condições de cada cirurgia e do paciente. Em relação ao possível risco de hemorragias, a literatura não indica a existência de complicações relevantes com o uso de heparina de baixo peso molecular, porém, o seu uso agrega um valor maior no tratamento do paciente. Entretanto, ainda que os resultados positivos da tromboprofilaxia sejam notórios, muitos médicos ainda não fazem uso dessa estratégia.

Tendo em vista os aspectos observados, entende-se que as maiores dificuldades para que o uso da profilaxia seja um método difundido nas cirurgias plásticas estão relacionados ao custo desses medicamentos visto que, na maioria das vezes não são oferecidos de forma gratuita. Além disso, os principais motivos que levam a subutilização desse processo preventivo e que, consequentemente, acarretam a um aumento de mortes que poderiam ser evitadas são o desconhecimento do procedimento e a inaptidão para a administração do tratamento pelo paciente. Vale lembrar ainda que cada caso é único, e o risco de tromboembolismo venoso depende da condição do paciente e do procedimento realizado.

\section{Referências}

Abreu, A. P. A. (2016). Risco tromboembolítico: etiopatogenia e profilaxia em pacientes mastectomizadas submetidas a reconstrução mamária. Instituto de Ciências Biomédicas Abel Salazar, Porto.

Amaral, C., Pereira, L. G., Moreto, A., Sá, A. C., \& Azevedo, A. (2016). Estudo de tromboembolismo venoso no pós operatório - risco e mortalidade por especialidade cirúrgica. Revista Portuguesa de Cardiologia, 36 (9), 609-616.

Amaral, C., Reis, J., Guimarães, L., Sá, A. L., Moreto, A., Araújo, F., Guimarães, M., Felicíssimo, P., Teixeira, J., Fonseca, C., \& Miranda, L. (2014). Recomendações perioperatórias para profilaxia do tromboembolismo venoso no doente adulto. Rev Soc Port Anestesiol, 23 (3), $62-75$.

Anger, J., Baruzzi, A. C. A., \& Knobel, E. (2003). Um protocolo de prevenção de trombose venosa profunda em cirurgia plástica. Revista da Sociedade Brasileira de Cirurgia Plástica, 18 (1), 47-54.

Barros, D. A. S. N. (2021). Prevenção de tromboembolismo venoso em cirurgia plástica: revisão de literatura. Escola de Saúde Pública do Estado do Ceará, Ceará, Brasil.

Cunca-Pardo, J., Gallardo, G. R., Camarena, L. C., Bulnes, L. C., \& Alvear, G. L. (2019). Searching for the Best Way to Assess the Risk of Thrombosis in Aesthetic Plastic Surgery; The Role of the Caprini/Pannucci Score. Aesthetic Plastic Surgery, 43 (5), 1387-1395.

Drouin, L., Pistorius, M. A., Lafforgue, A., Gohou, C. N., Richard, A., Connault, J., \& Espitia, O. (2018). La Revue de Médecine Interne, 40 (1), 1-17.

Hinrichsen, S. L., Mouzinho, D., Carvalho, E., Oliveira, C. L. F., Possas, L., \& Vilela, T. A. S. (2014). Implementação do programa de prevenção de tromboembolismo venoso (TEV): um estudo piloto. Revista ACRED, 4 (7), 1-9.

Hoyos, M. B. L. (2021). Tromboembolismo pulmonar decorrente de tromboflebites venosas superficiais em membros superiores pós-cirurgia plástica mamária estética: relato de 3 casos. Revista Brasileira de Cirurgia Plástica, 36 (1), 85-90.

Iorio, M. L., Venturi, M. L., \& Davison, S. P. (2015). Diretrizes práticas para quimioprofilaxia de tromboembolismo venoso em cirurgia plástica eletiva. Cosmetic, 135 (2), 413-423.

Justino, T. A., Varoni, A. C. C., \& Duz, G. L. (2018). Tromboembolismo venoso (TEV) em abdominoplastias: um protocolo de prevenção. Revista Brasileira de Cirurgia Plástica, 33 (1), 33-38.

Mittal, P., Heuft, T., Richter, D. F., \& Wiedner, M. (2020). Profilaxia de tromboembolismo venoso (TEV) após abdominoplastia e lipoaspiração: uma revisão da literatura. Aesthetic Plastic Surgery, 44 (2), 473-482.

Murphy, R. X., Alderman, A., Gutowski, K., Kerrigan, C., Rosolowski, K., Schechter, L., Schmitz, D., \& Wilkins, E. (2012). American Society of Plastic Surgeons, 130 (1), 168-175.

Paiva, R. A., Chadraqui, J., Paiva, R, Machado, B. A., Fishdick, H., \& Pitanguy, I. (2015). Protocolo de prevenção de tromboembolismo venoso em cirurgia plástica: resultados em 2759 pacientes no Instituto Ivo Pitanguy. Revista Brasileira de Cirurgia Plástica, 30 (2), $242-249$.

Paiva, R. A., Pitanguy, I., Amorim, N. F. G., Shdick, R. B. H. A., \& Holanda, T. A. (2010). Tromboembolismo venoso em cirurgia plástica: protocolo de prevenção na Clínica Ivo Pitanguy. Revista Brasileira de Cirurgia Plástica, 25 (4), 583-588.

Pannucci, C. J. (2016). Evidence-based recies for venous thromboembolism prophylaxis: a practical safety guide. American Society of Plastic Surgeons, 139 (2), 520- 533 .

Reis Neto, R. S., Varoni, A. C. C., Duz, G. L., Soler, T. S., \& Abreu, A. M. R. (2019). Fenômenos tromboembólicos associados a mamoplastia no Serviço de Cirurgia Plástica do Hospital PUC-Campinas. Revista Brasileira de Cirurgia Plástica, 34 (3), 310-314.

Roça, G. B., Bernert, B. F., \& Freitas, R. S. (2018). Rivaroxabana para profilaxia de tromboembolismo venoso em abdominoplastia após grande perda ponderal: 396 casos. Revista Brasileira de Cirurgia Plástica, 34 (2), 268-273. 
Research, Society and Development, v. 10, n. 11, e267101119687, 2021

(CC BY 4.0) | ISSN 2525-3409 | DOI: http://dx.doi.org/10.33448/rsd-v10i11.19687

Santos, G. S. S., \& Oliveira, D. F. (2017). Os entraves da profilaxia da trombose venosa profunda: uma revisão integrativa de literatura. Revista Acreditação, 7 (13), 77-96.

Swanson, E. (2017). Preocupações com relação ao uso de anticoagulantes orais (rivaroxabana e apixabana) para profilaxia de tromboembolismo venoso em pacientes de cirurgia plástica. Aesthetic Plastic Surgery, 36 (8), 262-264.

Valente, D. S., Carvalho, L. A., Zanella, R. K., \& Valente, S. (2014). Tromboembolismo venoso após cirurgia plástica estética eletiva: um estudo prospectivo longitudinal em 1.254 pacientes. Plastic Surgery International, 2014 (1). 